MARCOS ROSA DOS SANTOS

\title{
METODOLOGIAS PARA EXECUÇÃO DE MANOBRAS DE PARALELISMO EM REDES AÉREAS DE DISTRIBUIÇÃO NA OPERAÇÃO DE TEMPO REAL
}

Tese apresentada à Escola Politécnica da Universidade de São Paulo para obtenção do título de Doutor em Engenharia 
MARCOS ROSA DOS SANTOS

\section{METODOLOGIAS PARA EXECUÇÃO DE MANOBRAS DE PARALELISMO EM REDES AÉREAS DE DISTRIBUIÇÃO NA OPERAÇÃO DE TEMPO REAL}

Tese apresentada à Escola Politécnica da Universidade de São Paulo para obtenção do título de Doutor em Engenharia

Área de concentração:

Sistemas de Potência

Orientador:

Prof. Dr. Carlos Eduardo de Morais Pereira. 
Este exemplar foi revisado e corrigido em relação à versão original, sob responsabilidade única do autor e com a anuência de seu orientador.

São Paulo, de maio de 2013.

Assinatura do autor

Assinatura do orientador

FICHA CATALOGRÁFICA

Santos, Marcos Rosa dos

Metodologias para execução de manobras de paralelismo em redes aéreas de distribuição na operação de tempo real / M.R. dos Santos. Versão corr. -- São Paulo, 2013.

$186 \mathrm{p}$.

Tese (Doutorado) - Escola Politécnica da Universidade de São Paulo. Departamento de Engenharia de Energia e Automação Elétricas.

1. Redes de distribuição de energia elétrica 2. Tempo-real (Operação) 3. Paralelismo de alimentadores I. Universidade de São Paulo. Escola Politécnica. Departamento de Engenharia de Energia e Automação Elétricas II. t. 
À Deus 


\section{AGRADECIMENTOS}

À Deus.

À minha família.

Ao Prof. Dr. Carlos Eduardo de Morais Pereira, pela orientação dispensada no decorrer do trabalho.

Aos Profs. Drs. Hernan Pietro Schimidt e Giovanni Manassero Jr., pelas sugestões e comentários apresentados na qualificação.

À AES Eletropaulo por permitir testes na rede aérea primária de distribuição.

Aos amigos Sandro Zanata e Jairo Lima da Costa pela colaboração na revisão do texto e montagem do trabalho.

Às demais pessoas que, direta ou indiretamente, contribuíram na execução deste trabalho. 
Talvez eu seja enganado inúmeras vezes. Mas não deixarei de acreditar que, em algum lugar, alguém merece a minha confiança.

(Aristóteles) 


\section{SUMÁRIO}

1. Introdução e Revisão Bibliográfica ............................................26

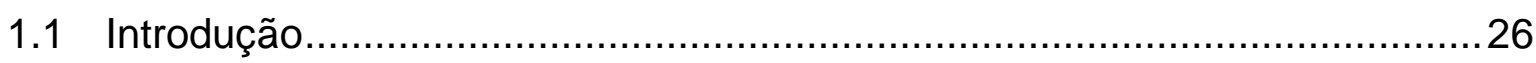

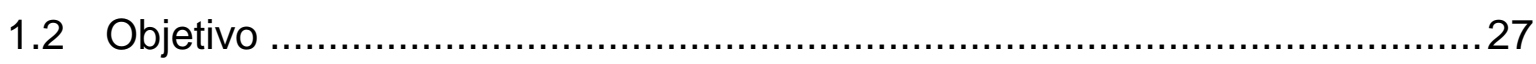

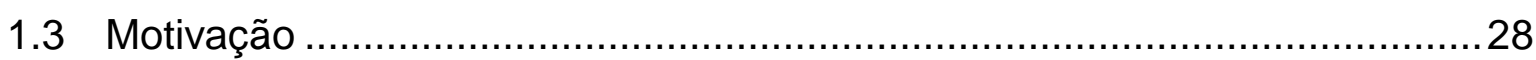

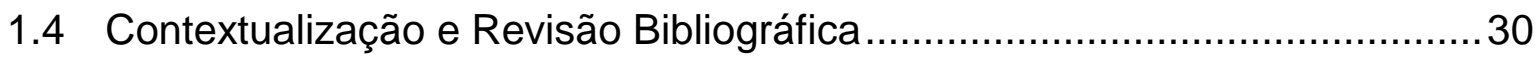

2. Metodologia e Modelagem .....................................................46

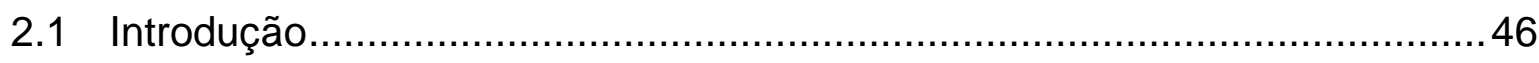

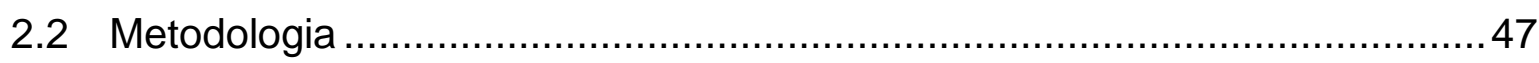

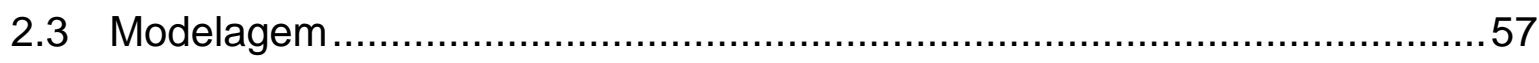

3. Simulações e Resultados Reais...............................................67

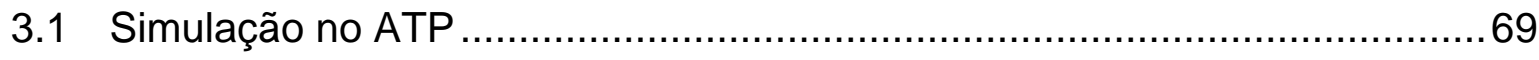

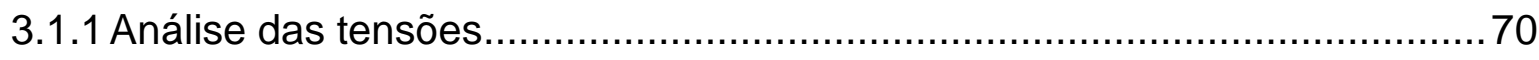

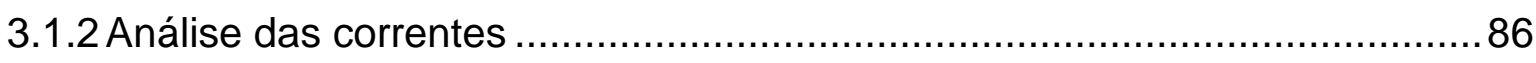

3.2 Medições reais das Manobras Realizadas ………...................................96

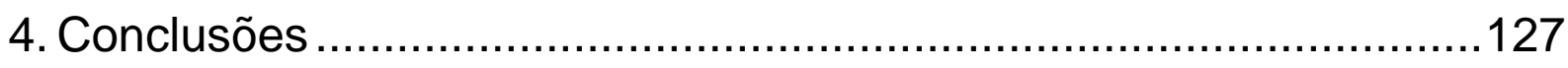

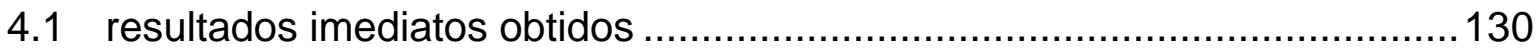

4.2 Propostas de novos trabalhos ……....................................................... 132

Anexo A - Proteção e cálculo de curto-circuito..................................133

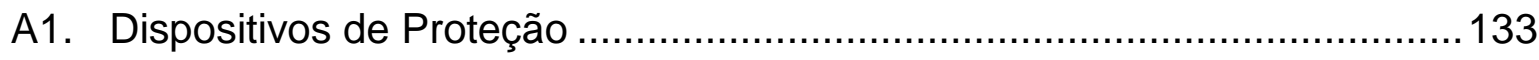

A2. Cálculo de Curto-Circuito para os Alimentadores BRU-109 e TAM114.

Anexo B - Carregamento das Subestações Edgard de Souza e Anhanguera .................................................................. 145

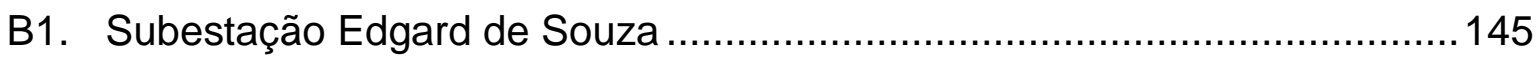


B2. Subestação Anhanguera

Anexo C - Equipamentos e Acessórios da Rede Aérea Primária

C1. Postes

C2. Estruturas Básicas Classe $15 \mathrm{kV}$ - Rede Convencional. 155

C3. Rede Compacta 159

C4. Rede com Cabos Pré-Reunidos 162

C5. Travessias 164

C6. Afastamentos Mínimos Padrão ABNT 166

C7. Condutores 168

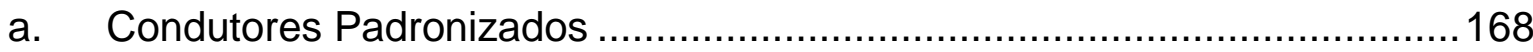

b. Dimensionamento de Condutores ..........................................................169

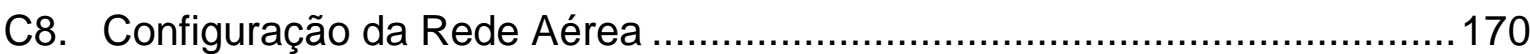

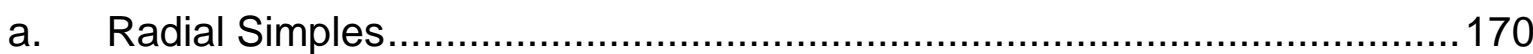

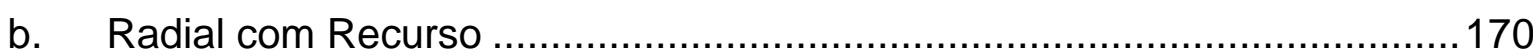

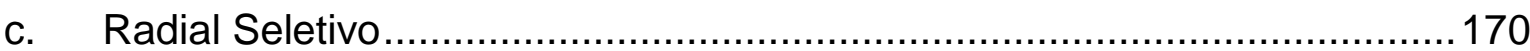

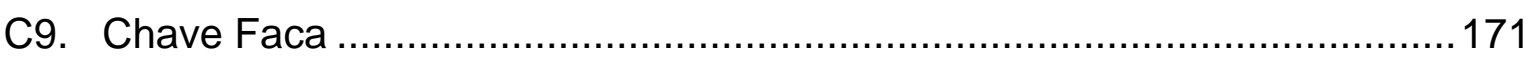

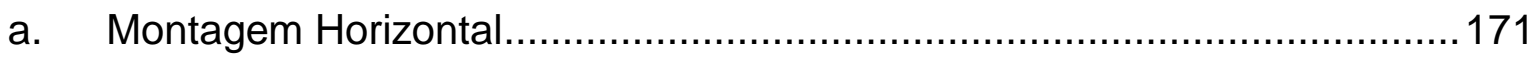

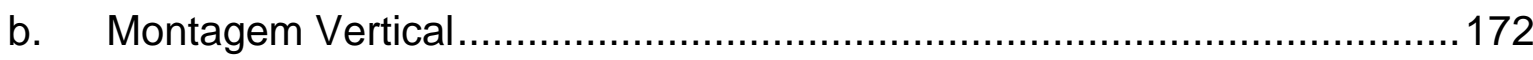

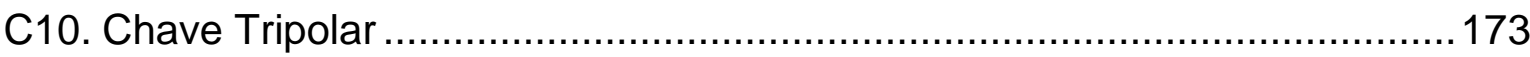

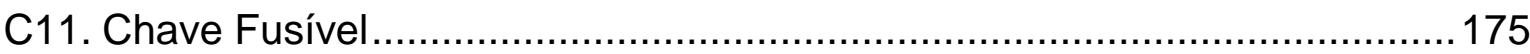

C12. Saída de Circuitos em Estações Transformadoras de Distribuição .............176

a. Saída Convencional de Alimentador....................................................176

b. Saída Através de Pré-Reunido ................................................................177

Anexo D - Parâmetros Elétricos de Equipamentos e Estruturas .........178

Anexo E - Dados Técnicos do Medidor Elspec Blackbox G4500 ........181

Referências Bibliográficas ............................................................185 


\section{LISTA DE FIGURAS}

Figura 1.1 - Diagrama esquemático da transmissão brasileira.

Figura 1.2 - Demanda do Sistema Interligado Nacional brasileiro em 2011 e 2012 32

Figura 1.3 - Demanda da região Sudeste do Brasil em 2011 e 2012. 33

Figura 1.4 - Demanda do sistema de subtransmissão da AES Eletropaulo abril/2012. 34

Figura 1.5 - Representação simplificada dos estados de um AL pós-falta. 37

Figura 1.6 - Diagrama unifilar representando transferência de carga entre dois alimentadores. 39

Figura 1.7 - Diagrama unifilar simplificado para transferência de carga entre dois alimentadores.

Figura 1.8 - Esquema simplificado de fechamento em anel entre dois alimentadores

Figura 1.9 - Transformadores de estações transformadoras de distribuição adjacentes, com mesmas características.

Figura 1.10 - Curvas das capacidades de curto-circuito versus a distância entre a estação transformadora de distribuição e o ponto do alimentador em falta.

Figura 2.1 - Algoritmo para fechamento em anel entre dois alimentadores. 48

Figura 2.2 - Diagrama unifilar das S/Es, LTAs e ETDs. .50

Figura 2.3 - Diagrama unifilar da S/E Edgard de Souza. 51

Figura 2.4 - Diagrama unifilar da S/E Anhanguera. 52

Figura 2.5 - Carregamentos secundários dos transformadores das S/Es Edgard de Souza e Anhanguera. 53 
Figura 2.6 - Diagrama unifilar da ETD Barueri.

Figura 2.7 - Diagrama unifilar da ETD Tamboré: a) Transformador 2, alimentando barras 2 e 4 de 13,8 kV; b) Transformador 1, alimentando barras 1 e 3 de 13,8 kV 55

Figura 2.8 - Diagrama unifilar dos alimentadores vistos no xOMINI Maps: a) BRU-109, e; b) TAM-114 58

Figura 2.9 - Diagrama dos circuitos BRU-109 x TAM-114 de 13,8 kV xOMINI Maps 59

Figura 2.10 - Carregamento do alimentador BRU-109 - Dados do Cymdist. 61

Figura 2.11 - Carregamento do alimentador TAM-114 - Dados do Cymdist. 62

Figura 2.12 - Carregamento de trecho do TAM-114, alem seccionadora 40343 - Cymdist. 63

Figura 2.13 - Modelagem dos alimentadores BRU-109 e TAM-114 no ATP:

a) Representação das S/Es, ETDs e modelo do acoplamento entre as S/Es; b) Seccionadoras de fechamento e abertura do anel entre as S/Es. 65

Figura 2.14 - Esquema simplificado de fechamento em anel do tipo IX, entre dois alimentadores. 66

Figura 3.1 - Medidor de qualidade de energia G4500 Blackbox da ELSPEC®. 67

Figura 3.2 - Tensão de fase no transformador 2 da ETD Tamboré - ATP. .70

Figura 3.3 - Tensão de linha no transformador 2 da ETD Tamboré - ATP. 71

Figura 3.4 - Tensões de linha nos primários dos bancos de transformadores das S/Es Edgard de Souza e Anhanguera - ATP.... .72

Figura 3.5 - Tensão de linha no primário do transformador 2 da ETD Barueri - ATP .73 
Figura 3.6 - Tensão de linha no primário do transformador 2 da ETD Tamboré - ATP

Figura 3.7 - Tensão de linha no secundário do transformador 2 da ETD Barueri - ATP

Figura 3.8 - Tensão de linha no secundário do transformador 2 da ETD Tamboré - ATP. .77

Figura 3.9 - Tensões nos terminais das seccionadoras 43286 e 40343 ATP 78

Figura 3.10 - Tensões máximas nos terminais da seccionadora de vis-à-vis 43286 antes do fechamento - ATP. 79

Figura 3.11 - Tensões eficazes nos terminais da seccionadora de vis-à-vis 43286 no momento do fechamento - ATP. 80

Figura 3.12 - Tensões pré-manobra de abertura nos terminais da seccionadora 40343 - ATP.

Figura 3.13 - Chave LoadBuster para manobra de seccionadoras sob carga. 82

Figura 3.14 - Tensões de fase pré-manobra de fechamento da seccionadora de vis-à-vis 43286 - ATP 83

Figura 3.15 - Tensões pós-manobra de abertura da seccionadora 40343 ATP. 84

Figura 3.16 - Tensões pré e pós manobras de fechamento em anel, a jusante da seccionadora 40343 e do secundário do transformador 2 da ETD Tamboré - ATP. 85

Figura 3.17 - Carregamentos dos alimentadores: a) BRU-109, e; b) TAM114, de $13,8 \mathrm{kV}$ - ATP. 87

Figura 3.18 - Carregamentos dos alimentadores BRU-109 e TAM-114 referidos ao primário dos bancos de transformadores das S/Es Anhanguera e Edgard de Souza - ATP. 
Figura 3.19 - Carregamentos dos alimentadores BRU-109 e TAM-114 referidos nas LTAs Anhanguera-Mutinga e Edgard de Souza-Mutinga de $88 \mathrm{kV}-\mathrm{ATP}$.

Figura 3.20 - Correntes pré e pós manobras de fechamento da chave de visà-vis 43286 - ATP.

Figura 3.21 - Correntes pré e pós manobras das seccionadoras 43286 e 40343 - Medições nas estações transformadoras de distribuição.

Figura 3.22 - Corrente pré e pós manobras de abertura da seccionadora monopolar 40343 - ATP. 94

Figura 3.23 - Tensão nas barras das S/Es Anhanguera e Edgard de Souza. 100

Figura 3.24 - Tensões rms dos secundários dos transformadores das ETDs Barueri e Tamboré durante as manobras de fechamento e abertura do anel. 104

Figura 3.25 - Correntes rms dos alimentadores BRU-109 e TAM-114 durante as manobra de fechamento e abertura do anel..... 105

Figura 3.26 - Tensões e correntes rms para os secundários dos transformadores e para os alimentadores envolvidos nas manobras de fechamento em anel. 106

Figura 3.27 - Forma de onda com os mínimos e máximos das correntes dos alimentadores BRU-109 e TAM-114, Fase 1.

Figura 3.28 - Forma de onda com os mínimos e máximos das correntes dos alimentadores BRU-109 e TAM-114, Fase 2.

Figura 3.29 - Forma de onda com os mínimos e máximos das correntes dos alimentadores BRU-109 e TAM-114, Fase 3. 108

Figura 3.30 - Forma de onda da corrente do neutro dos alimentadores BRU109 e TAM-114. 109

Figura 3.31 - Forma de onda das correntes dos alimentadores BRU-109 e 
Figura 3.32 - Detalhe das correntes dos alimentadores no momento do fechamento do anel entre as S/Es Anhanguera e Edgard de Souza.

Figura 3.33 - Forma de onda das correntes dos alimentadores BRU-109 e TAM-114, com a ampliação do ponto $A$.

Figura 3.34 - Forma de onda das correntes dos alimentadores BRU-109 e TAM-114, com a ampliação do ponto $A 1$.

Figura 3.35 - Detalhe das correntes dos alimentadores no momento da abertura do anel entre as S/Es Anhanguera e Edgard de Souza.

Figura 3.36 - Forma de onda das correntes dos alimentadores BRU-109 e TAM-114, com a ampliação do ponto B.

Figura 3.37 - Forma de onda das correntes dos alimentadores BRU-109 e TAM-114, com a ampliação do ponto B1. 116

Figura 3.38 - Correntes dos alimentadores no momento do fechamento do anel entre as S/Es.

Figura 3.39 - Forma de onda das correntes dos alimentadores BRU-109 e TAM-114, com a ampliação do ponto C. 118

Figura 3.40 - Forma de onda das correntes dos ALs BRU-109 e TAM-114, com a ampliação do ponto $C 1$

Figura 3.41 - Forma de onda das correntes dos alimentadores com a ampliação do ponto $\mathrm{D}$ 120

Figura 3.42 - Correntes dos alimentadores no momento da abertura do anel entre as S/Es Anhanguera e Edgard de Souza.

Figura 3.43 - Forma de onda das correntes: a) Ponto $E$, ampliado para os ALs BRU-109 e TAM-114; b) Ponto E1 para BRU-109, e; c) Ponto E1 para TAM-114. 
Figura 3.44 - Forma de onda das correntes: a) Ponto $F$, ampliado para os ALs BRU-109 e TAM-114; b) Ponto F1 para o BRU-109, e; c) Ponto F1 para o TAM-114 124

Figura 3.45 - Forma de onda das correntes: a) Ponto $\mathrm{H}$, ampliado para os ALs BRU-109 e TAM-114: b) Ponto H1 para o BRU-109, e; c) Ponto H1 para o TAM-114 126

Figura A.1 - Diagrama unifilar simplificado de proteção. 133

Figura A.2 - Diagrama trifilar simplificado de proteção. 134

Figura A.3 - Relé de sobrecorrente CDG com disco de indução: a) Esquema de funcionamento; b) Detalhes do relé. 136

Figura A.4 - Corrente circulando em um alimentador para condição normal de operação. 136

Figura A.5 - Tipos de faltas encontradas na rede: a) curto-circuito monofásico, fase-terra; b) curto-circuito dupla fase, e; c) curto-circuito trifásico. 138

Figura A.6 - Amplitudes e ângulos das correntes de curto-circuito trifásico para o alimentador BRU-109 próximo à ETD Barueri.

Figura A.7 - Amplitudes e ângulos das correntes de curto-circuito fase-terra para o alimentador BRU-109 no ponto mais distante da ETD Barueri.

Figura A.8 - Amplitudes e ângulos das correntes de curto-circuito trifásico para o alimentador TAM-114 próximo à ETD Tamboré 143

Figura A.9 - Amplitudes e ângulos das correntes de curto-circuito fase-terra para o alimentador TAM-114 no ponto mais distante da ETD Tamboré. 144

Figura B.10 - Demandas máximas diárias da S/E Edgard de Souza, período julho/2011 - Patamares de cargas leve/média e pesada. 146

Figura B.11 - Demandas máximas da S/E Edgard de Souza para o mês de julho de 2011. 
Figura B.12 - Curvas diárias das demandas da S/E Edgard de Souza para o mês de julho de 2011

Figura B.13 - Demandas máximas diárias da S/E Anhanguera para o mês de julho de 2011 - Patamares de cargas leve/média e pesada.

Figura B.14 - Demandas máximas da S/E Anhanguera para o mês de julho de 2011

Figura B.15 - Curvas diárias das demandas da S/E Anhanguera para o mês de julho de 2011

Figura B.16 - Carregamentos dos bancos de transformadores da S/E Edgard de Souza em 13/07/2011. 152

Figura B.17 - Carregamentos dos bancos de transformadores da S/E Anhanguera em 13/07/2011. 152

Figura C.18 - Estrutura básica classe 15 kV. 153

Figura C.19 - Estrutura básica classe 15 kV: a) Estrutura M1; b) Estrutura M4. 155

Figura C.20 - Estrutura básica classe 15 kV: a) Estrutura M3; b) Estrutura B1. 156

Figura C.21 - Estrutura básica classe 15 kV: a) Estrutura B3; b) Estrutura B4. 156

Figura C.22 - Estrutura classe $15 \mathrm{kV}$ tipo RC1. 157

Figura C.23 - Estrutura classe $15 \mathrm{kV}$ tipo RC2. 158

Figura C.24 - Estrutura classe $15 \mathrm{kV}$ tipo RC3. 158

Figura C.25 - Estrutura classe $15 \mathrm{kV}$ tipo RC4. 159

Figura C.26 - Estrutura classe $15 \mathrm{kV}$ tipo RC5. 159

Figura C.27 - Estrutura classe $15 \mathrm{kV}$ tipo RC6. 160 
Figura C.28 - Estrutura classe 15 kV tipo RC3 Dupla. 160

Figura C.29 - Estrutura classe 15 kV tipo RCEL...............................................161

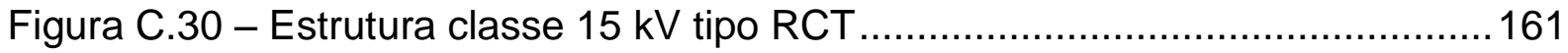

Figura C.31 - Estrutura classe $15 \mathrm{kV}$ - Suporte simples PRE1 …....................... 162

Figura C.32 - Estrutura classe 15 kV - Suporte duplo PRE2 …...........................163

Figura C.33 - Estrutura classe $15 \mathrm{kV}$ - Ancoragem simples PRE3 ......................163

Figura C.34 - Estrutura classe 15 kV - Ancoragem dupla PRE4 .........................163

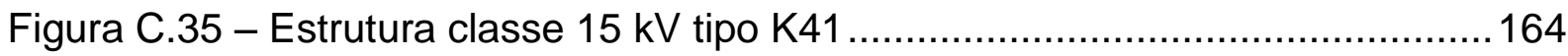

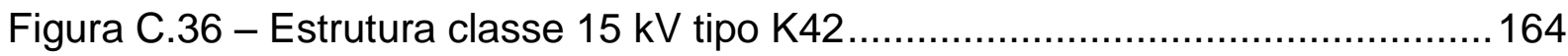

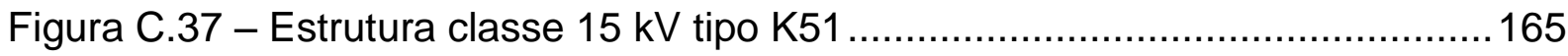

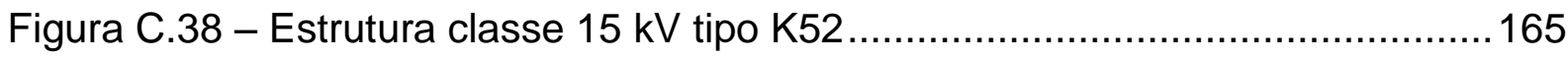

Figura C.39 - Montagem horizontal de chave faca........................................... 171

Figura C.40 - Montagem vertical de chave faca. ............................................ 172

Figura C.41 - Chave tripolar - vista frontal. .................................................173

Figura C.42 - Chave tripolar - vista lateral. ................................................ 174

Figura C.43 - Chave tripolar: a) Operação manual, e; b) Automatizada..................174

Figura C.44 - Padrão de instalação de chave fusível. ........................................175

Figura C.45 - Saída convencional de alimentador aéreo primário.........................176

Figura C.46 - Saída de alimentador utilizando cabo pré-reunido..........................177

Figura E.47 - Medidor de qualidade de energia Blackbox G4500 ......................181

Figura E.48 - Diagrama de blocos do tratamento dos dados do Blackbox G4500 pelo PQSCADA. 


\section{LISTA DE TABELAS}

Tabela 2.1 - Possibilidades de manobras de paralelismos entre alimentadores

Tabela 2.2 - Comprimentos dos cabos condutores dos alimentadores BRU109 e TAM-114.

Tabela 2.3 - Carregamento dos alimentadores calculados pelo software Cymdist.

Tabela 3.1 - Dados de sincronização e amostragem do medidor Elspec Blackbox G4500. 68

Tabela 3.2 - Tempos de abertura e fechamento das chaves seccionadoras 43286 e 40343, utilizados na parametrização do aplicativo ATP.

Tabela 3.3 - Transformadores utilizados nas estações da AES Eletropaulo com ou sem reguladores de tensão. 75

Tabela 3.4 - Características elétricas das seccionadoras de distribuição classe $15 \mathrm{kV}$.

Tabela 3.5 - Cargas dos alimentadores TAM-114 e BRU-109 no momento das manobras - SSC.

Tabela 3.6 - Tensões e correntes dos transformadores das estações transformadoras de distribuição.

Tabela 3.7 - Carregamentos e capacidades dos alimentadores BRU-109 e TAM-114

Tabela 3.8 - Carregamentos e capacidades dos transformadores das ETDs Barueri e Tamboré. 102

Tabela 4.1 - Carga do trecho do alimentador TAM-114, entre seccionadoras 40343 e 43286 . 128

Tabela 4.2 - Capacidade de curto-circuito das barras secundárias dos 
Tabela C.3 - Capacidade dos postes em função das alturas: a) Postes de concreto; b) Postes de madeira; c) Postes especiais. 154

Tabela C.4 - Distâncias entre condutores de um mesmo circuito 166

Tabela C.5 - Distâncias mínimas das partes energizadas à fase ou a terra em pontos fixos. 166

Tabela C.6 - Distâncias entre condutores de circuitos diferentes. 166

Tabela C.7 - Distâncias entre os condutores e o solo. 167

Tabela C.8 - Dimensionamento de condutores. 169

Tabela C.9 - Dados técnicos característicos de uma chave faca. 175

Tabela D.10 - Parâmetros elétricos dos bancos de transformadores das S/Es Edgard de Souza e Anhanguera. 178

Tabela D.11 - Parâmetros elétricos das linhas de subtransmissão aéreas. 179

Tabela D.12 - Parâmetros elétricos dos transformadores das ETDs Barueri e Tamboré. 179

Tabela D.13 - Parâmetros elétricos dos alimentadores aéreos primários BRU-109 e TAM-114, ambos de 13,8 kV. 180

Tabela E.14 - Dados gerais do medidor Blackbox G4500. 182

Tabela E.15 - Dados de entrada de tensão e corrente do medidor Blackbox G4500 182

Tabela E.16 - Sincronização e amostragem do medidor Blackbox G4500. 183

Tabela E.17 - Faixas de medição do medidor Blackbox G4500. 183

Tabela E.18 - Detecção de transitórios de tensão do medidor Blackbox G4500. 


\section{LISTA DE ABREVIATURAS E SIGLAS}

A2: classe de tensão de fornecimento de energia elétrica $(88 \mathrm{kV})$

AC: corrente alternada

AL: alimentador aéreo primário

ALT: alimentador com limitação térmica

ANEEL: Agência Nacional de Energia Elétrica

ANH: Anhanguera

AQT: alimentador com queda de tensão limitada

ARSESP: Agência Reguladora de Saneamento e Energia do Estado de São

Paulo

ATP: programa para cálculo numérico de transitórios eletromagnéticos em sistemas

de potência

AZ: fase azul

BC: banco de capacitores instalado ao longo do AL

BCA: banco de capacitores instalado na barra da ETD

BJA: Bom Jardim

BR: fase branca

BRU: Barueri

CCC: capacidade de curto circuito

CF: chave faca

CH SECC: chave seccionadora

$\mathrm{CH}$ VIS: chave seccionadora de vis-à-vis.

COD: centro de operação da distribuição

CSPE: Comissão de Serviços Públicos de Energia

CTEEP: Companhia de Transmissão de Energia Elétrica Paulista 
CYMDIST: Distribution System Analysis

DEC: duração equivalente de interrupção por unidade consumidora

DIC: duração de interrupção individual por unidade consumidora

DJ: disjuntor

DMIC: duração máxima de interrupção contínua por unidade consumidora

DMS: distribution management system

EBC: estação de banco de capacitores

EOC: estação de chaves classe $88 / 138$ kV, na subtransmissão

EPC: equipamento de proteção coletivo

EPI: equipamento de proteção individual

ETD: estação transformadora de distribuição

EMS: energy management system

ESD: estação do sistema de distribuição

ESO: Edgard de Souza

FALTA: condição da rede durante a falta

FEC: frequência equivalente de interrupção por unidade consumidora

FIC: frequência de interrupção individual por unidade consumidora

FUNDAMENTAL: componente de $60 \mathrm{~Hz}$ do sinal com harmônicas

GIS: geographic information systems

GPS: "Global Positioning System", sistema de localização geográfica e de referência de tempo global

i: corrente elétrica

IEO: instrução específica de operação

IMPEDÂNCIA-SÉRIE: modelo de linha que desconsidera a capacitância da linha e o efeito do comprimento

LB: Loadbuster, bastão de manobras com câmara de extinção de arco voltaico 
LI: linha de interligação

LINE CONSTANTS: rotina do programa ATP para cálculo de parâmetros de linhas de transmissão

LT: linha de transmissão

LTA: linha de transmissão aérea

LTS: linha de transmissão subterrânea

MATLAB: programa de processamento numérico e geração de gráficos

MCM: milhar circular mil

MPT: manual de procedimento técnico

MODELS: rotina do programa ATP similar a linguagens de programação de alto nível, e que permite o modelamento de componentes da rede, geração de arquivos de saída e interfaceamento com a linguagem "C"

MUT: Mutinga

MVA: mega volt ampèr

OMS: outage management system

ONS: Operador Nacional do Sistema

OTR: operação em tempo real

PRÉ-FALTA: condição da rede antes da ocorrência de falta PRI: Pirituba

PRODIST: procedimentos de distribuição de energia elétrica

PÓS-FALTA: condição da rede durante a falta

pu: por unidade

RA: religadora automática

RMS: root mean square (valor médio eficaz para grandezas elétricas)

$\mathrm{RP}$ : regime permanente

RRA: relé de religamento automático

S/E: subestação de transmissão de energia 
SCADA: supervisory control and data acquisition

SECC: seccionadora

SEP: sistema elétrico de potência

SIN: sistema interligado nacional

SINAP: Sistema de Análise Integrada AT/MT/BT

SPOT NETWORK: rede distribuição curta e local, comumente utilizados em sistemas reticulados subterrâneos

SSC: sistema de supervisão e controle

TA: transferência automática

TAM: Tamboré

TAP: derivação elétrica no enrolamento de um TR, podendo ser no primário ou no secundário

$\mathrm{TCH}$ : torre de chaves seccionadoras

THD: taxa de distorção harmônica total

TR: transformador

TRIP: comando de desligar

U: tensão elétrica

V: tensão elétrica

VA: volt-ampèr

Vef: tensão eficaz

VIS-À-VIS: fronteira entre dois alimentadores onde é possível o fechamento em anel

$\pi$-corrigido: modelo de linha que considera a capacitância da linha e o efeito do comprimento

$\pi$-nominal: modelo de linha que considera a capacitância da linha, mas despreza o efeito do comprimento 


\section{RESUMO}

Este trabalho tem por objetivo apresentar novos desenvolvimentos e a evolução do conceito de manobras de paralelismo entre alimentadores aéreos primários de distribuição, bem como os efeitos eletromagnéticos provocados por manobras em tempo real, visando a segurança operacional dos equipamentos e operadores de campo.

São apresentadas propostas para fechamento de subestações transformadoras de transmissão em anel, através de alimentadores aéreos primários, além de algoritmo simplificado para a operação e despacho do sistema.

Uma nova metodologia de operação em tempo real foi desenvolvida através de algoritmo de viabilidade usando dados do SCADA (Supervisory Control and Data Acquisition). Esta metodologia de operação oferece algumas vantagens como o restabelecimento de cargas interrompidas, o isolamento de trechos defeituosos na rede de distribuição aérea, bem como o atendimento de serviços programados no sistema elétrico de potência.

Para essa metodologia é feita uma análise das grandezas elétricas e capacidades dos equipamentos envolvidos, bem como dos ajustes e parâmetros dos dispositivos de proteção, de forma a obter sucesso na operação da rede em tempo real.

O estudo foi baseado em manobras reais em alimentadores aéreos de distribuição da AES Eletropaulo. A validação do modelo que direcionou para o sucesso das manobras em tempo real foi baseada principalmente em simulações de transitórios eletromagnéticos, fluxo de carga e curto-circuito através dos programas ATP e CYMDIST. 


\section{ABSTRACT}

This work aims to present new developments and the evolution of the parallelism switch operation concept among distribution primary overhead feeders, as well as the electromagnetic effects caused by switch operations in real time, in order to keep field operators and equipments in safety.

Hereby are presented some proposals for closed-loop arrangement between two power transmission substations through overhead feeders and a simplified algorithm for the system's operation and dispatch as well.

A new real time operation methodology was developed through viability algorithm using the SCADA's (Supervisory Control and Data Acquisition) database. This operation methodology offers some advantages such as restoration of interrupted loads, isolation of the faulty section in the overhead distribution network as well as scheduled services.

For this methodology was made an analysis of the electric magnitudes and of the involved equipment capabilities, as well as the protection devices' adjustments and parameters, so as to obtain successful in real time operation.

The study was based on actual switch operations over the distribution overhead feeders of AES Eletropaulo. The validation of the model that drove to the real time switch operations success was mainly based on electromagnetic transients simulations, load flow and short-circuit in ATP (Alternative Transient Program) and CYMDIST softwares. 


\section{ESTRUTURA DA TESE}

A tese está estruturada da seguinte forma:

\section{Capítulo 1}

Contém a introdução e a revisão bibliográfica sobre os tópicos mais relevantes de operação de redes aéreas primárias, dos transitórios eletromagnéticos provocados por manobras em tempo real, além dos efeitos físicos em componentes e equipamentos da rede em regime permanente quando dois alimentadores de diferentes subestações são fechados em anel.

\section{Capítulo 2}

Apresenta a metodologia empregada para desenvolvimento do novo algoritmo de fechamento em anel entre subestações de energia, realizado através do paralelismo entre dois alimentadores aéreos primários de distribuição. Também apresenta a modelagem dos alimentadores, transformadores e estações transformadoras de distribuição.

\section{Capítulo 3}

Neste capítulo são apresentadas as simulações realizadas no aplicativo ATP, os transitórios eletromagnéticos constatados no momento das manobras de formação do anel, além dos resultados obtidos em função dos testes reais realizados na rede aérea de distribuição da AES Eletropaulo.

\section{Capítulo 4}

Inclui a discussão e as conclusões, onde se busca esclarecer as vantagens e desvantagens do algoritmo desenvolvido. Contém propostas de novos trabalhos dentro dessa linha de pesquisa, dados técnicos de equipamentos e instrumentos utilizados, além das simulações de curto-circuito para os alimentadores estudados. 